\title{
CLAUDIO MAGRIS' Danubio EIN ÜBERSETZUNGSKRITISCHER TEXTVERGLEICH
}

Über den Fluß, an dessen Ufern man lebt, spricht man nicht. Wenn ein Regensburger über die Donau redet, dann muß das einen besonderen Grund haben, wie etwa ein Jahrhunderthochwasser oder das Buch von Claudio Magris Danubio, das, wenn man dem Bayerischen Rundfunk glauben kann, zu einem deutlichen Ansteigen der Zahl italienischer Besucher in der alten Reichsstadt Regensburg geführt hat ${ }^{1}$.

Ich möchte der Frage nachgehen, worin die Faszination begründet ist, die offensichtlich von diesem 1986 in italienischer Fassung erschienenen Buch ausgeht ${ }^{2}$, und ob die deutsche Übersetzung, die im Sommer 1988 veröffentlicht wurde ${ }^{3}$, in der Lage ist, diese Faszination weiterzugeben. Dabei werde ich die ebenfalls $1988 \mathrm{er}-$ schienenen Übersetzungen in spanischer (El Danubio) ${ }^{4}$ und französischer Sprache $(D a n u b e)^{5}$ in meine Überlegungen einbeziehen.

Die deutsche Ausgabe Donau trägt den Untertitel Biographie eines Flusses. Hieraus wird bereits deutlich, daß es sich nicht um einen Roman handelt ${ }^{6}$. Wir haben es mit einem - wenn auch nicht neuen, so doch seltenen - Genre zu tun, einer Mischung aus Essay, philosophischem Traktat und Reisebeschreibung?.

Eben weil es sich nicht "um eine Postkarten-Beschreibung handelt, sondern um Literatur, die erst aus der Distanz zeigt, was uns nahegeht “8 , stellte Magris bei seiner

1 Die ARD berichtete am 10.8.88 im Bayernstudio darüber: “...die Italiener haben Regensburg entdeckt. Auslöser für diesen Boom ist das Buch Danubio des Triester Professors Claudio Magris, das mit 400.000 verkauften Exemplaren in Italien ein absoluter Bestseller ist. Regensburg wird in einem Kapitel darin wahrhaft begeistert beschrieben. Offensichtlich so eindrucksvoll, daß sich südlich der Alpen immer mehr Kunstinteressierte auf den Weg machen, diese bayerische Stadt mit vielfältigen geschichtlichen Verbindungen nach Italien zu besuchen... Diese Begeisterung schlägt sich in der Statistik nieder. Um $15-20 \%$ stiegen in den letzten beiden Jahren die Übernachtungen der italienischen Touristen.in Regensburg - Tendenz steigend."

2 Garzanti Editore, Milano.

3 Hanserverlag, München.

4 Editorial Anagrama, Barcelona.

5 Gallimard, Paris.

6 Der Verlag wollte ursprünglich als Titel "Die Donau“ wählen, aber der Autor bestand auf "Donau" - ohne Artikel - mit dem Argument, das Buch sei kein Reiseführer, kein Baedeker, wie man vielleicht glauben möchte.

7 Am ehesten vergleichbar mit Magris' Werk scheint mir Carlo Levis Buch La doppia notte dei tigli. Was Reisebeschreibungen angeht, befindet sich Magris in guter Gesellschaft, man denke an Hugo, Stendhal oder Goethe.

8 Karl Birkenseer, "Donau" - eine Reise durch das Leben, in: Mittelbayerische Zeitung, 10.11.1988. 
Lesung in Regensburg auch nicht das Regensburgkapitel vor, sondern das Kapitel über den Zentralfriedhof in Wien, wo ein Herr Baumgartner als einer von drei Kleintierjägern damit beauftragt ist, "das rechte Gleichgewicht zwischen den hier mißbräuchlich und entgegen aller Vorschriften Lebenden herzustellen, um mit anderen Worten zu verhindern, daß es unter den Toten zu viele Lebewesen gibt, und diese, sofern sie sich auf dieser Welt allzu wohl fühlen und sich dabei noch vermehren sollten, alsbald in Tote zu verwandeln." $(214)^{9}$.

Dieses Kapitel gehört zu den wenigen, die sich gut zum Vorlesen eignen, denn über weite Strecken stellt Magris aufgrund der dargebotenen Informationsfülle und des hohen Stilniveaus höchste Ansprüche an die Aufmerksamkeit seiner Leser. Andererseits scheut er sich aber nicht, an geeigneter Stelle dialektale oder sogar vulgärsprachliche Elemente in Form von Zitaten einfließen zu lassen (worauf natürlich auch der Übersetzer Rücksicht nehmen muß, und was in den untersuchten Übersetzungen auch geschieht), so z.B. wenn er rückblickend seinen Lehrer Trani sagen läßt:

Te ghe podevi pensar prima ... perché no xe obligatorio aver fioi, adesso che te le ga fata, tientela, goditela, mantientela. (243)

Hättest eher dran denken sollen ... man muß nicht unbedingt Kinder haben, aber jetzt, wo du sie gemacht hast, behalt sie, freu dich an ihr [Tochter], sorg für sie. (268)

T'avais qu'à y penser avant, personne t'obligeait à avoir des gosses, maintenant que tu te l'es faite, tu te la gardes, tu te l'entretiens, et t'arrêtes de te plaindre! (281)

In der deutschen Fassung ist die Abweichung von der Hochsprache erkennbar, noch deutlicher wird sie jedoch in der französischen übersetzung (apostrophiertes $t^{\prime}$ statt $t u$, Verzicht auf ne, Verwendung von gosses statt enfants etc.). Weder im Deutschen noch im Französischen wird jedoch auf den Dialekt zurückgegriffen, sondern auf die Umgangssprache bzw. langue populaire. Die spanische Fassung läßt das Zitat unübersetzt und bringt in Klammern eine hochsprachliche übersetzung - die (unter stilistischen Gesichtspunkten) wohl schlechteste Lösung.

Ma il gioco delle coincidenze e delle concatenazioni che stringe l'universo ha deciso di differire l'esecuzione del fagiano assumendo un'altra veste, anch'essa però austriacamente burocratica; poco prima che il bersaglio diventi definitivamente sicuro ... compare un ansimante camioncino carico di foglie marce e di altri rifiuti, che i giardinieri del camposanto ... hanno raccolto nei viali e vengono a scaricare accanto a noi. Il fagiano, spaventato, si dilegua: il signor Baumgartner si concede un sonoro "merda!" ... (197)

Herr Baumgartner gestattet sich ein wohltönendes "Scheiße!" ... (218)

9 In Klammern die Seitenzahlen der jeweiligen Ausgabe. 
Il mondo, come dice Borges ${ }^{10}$, è reale, ma perché deve anche rompere tanto $\mathrm{i}$ coglioni? (340)

Die Welt, sagt Borges, ist wirklich, aber warum muß sie einem dermaßen auf den Sack gehen? (275)

El mundo, como dice Borges, es real, pero ¿por qué tiene también que tocar tanto los cojones? (293)

Le monde, comme dit Borgès, est bien réel, mais pourquoi faut-il aussi qu'il nous casse tellement les pieds? (391)

Die hohe Stilebene, auf der Magris sich aber offensichtlich am wohlsten fühlt, zeigt sich nicht nur in der häufigen Verwendung von Fremdwörtern und sonst eher selten gebrauchten Lexemen sowie unübersetzter Zitate oder fremdsprachlicher Termini, vor allem aus dem Französischen ("ogni nouvelle vague di giovani sperimentali ..." [332], dt.: "jede der verschiedenen nouvelles vagues junger experimentierfreudiger Autoren ..." [366], "homme à tout faire" [340]; dt. "homme à tout faire" [377]; "art d'après l'art" [344]; dt.: "art d'apres [sic!] l'art" [380]; "Gli studiosi sono severi con i mitografi sedotti dalle parole: Strabone et Pline ne sont pas excusables d'avoir dit que le meurtre d'Absirte se fit dans les Isles Absyrtides qui sont dans le golphe de Venise, dice il Dizionario di La Martinière" . [391]; dt.: "Die Gelehrten gehen mit den Mythographen, die sich von Wörtern verführen lassen, hart ins Gericht: Strabone et Pline ne sont pas ..., steht in dem Wörterbuch von La Martinière. " [433]), sondern wird auch in der bisweilen nicht unkomplizierten Syntax deutlich. Das Zusammentreffen beider Faktoren in Verbindung mit Magris' hintergründiger Metaphorik erfordert bisweilen ein mehrfaches Lesen oder besser "Studieren" einzelner Sätze. Er versteht es nicht nur, Parataxe und Hypotaxe geschickt zu verknüpfen, sondern ist auch darauf bedacht, den beim Lesen unweigerlich auftretenden Ermüdungserscheinungen durch das Einflechten trivialer Sachverhalte entgegenzuwirken. Er fordert Leser und Leserin, aber er überfordert sie nicht, und bewirkt dadurch - der Vergleich sei mir gestattet - einen Trainingseffekt wie beim Intervalltraining. schnitt.

Ein - wie ich meine - eindruckvolles Beispiel hierfür ist der folgende $\mathrm{Ab}$ -

10 Jorge Luis Borges, argentinischer Schriftsteller, Lyriker, Essayist.

11 Pinson de La Martin? 
Il Danubio scorre grande, e il vento della sera passa sui caffè all'aperto come il respiro di una vecchia Europa che forse è ormai ai margini del mondo e non produce ma solo consuma storia, come Francesca sta ora succhiando con la sua bella bocca il gelato, seduta nella pasticceria Gerbeaud, in piazza Vörösmarty, e guarda scivolare via la sua vita socchiudendo lievemente gli occhi sotto le sue famose ciglia, forse impercettibilmente aggrottate da quel frusciare del tempo. L'Europa è anche questo caffè, nel quale non siedono più gli Amministratori Delegati dello Spirito del Mondo ${ }^{12}$, ma tutt'al più i funzionari di qualche filiale subalterna, che non prendono ma eseguono decisioni, e qualche bella signora che fa chiacchierare di sé. (282)

Die Donau fließt großartig dahin, der Abendwind weht über die Cafés im Freien wie der Atem eines gealterten Europas, das nunmehr vielleicht am Rande der Welt sitzt und Geschichte nicht mehr hervorbringt, sondern nur noch konsumiert, so wie Francesca, die in der Konditorei Gerbeaud am. VörösmartyPlatz sitzt, mit ihrem schönen Mund an einen Eis lutscht und dabei mit halbgeschlossenen Augen unter ihren ruhmreichen Wimpern, die sich von diesem leichten Rauschen der Zeit unmerklich zusammengezogen haben, ihr eigenes Leben entgleiten sieht. Europa ist auch dieses Café, in dem nicht mehr die Geschäftsführer des Weltgeistes sitzen, sondern hlochstens die Vertreter einige[r] Unterfilialen, die keine Entscheidungen treffen, sondern sie ausführen, nebst einiger schöner Damen, die von sich reden machen. (311)

Magris führt uns zu einem Stelldichein mit zahlreichen Persönlichkeiten - im positiven wie im negativen Sinn -, aber auch unbedeutenden Menschen, die nur durch eine einzige Tat - im positiven wie im negativen Sinn - bekannt geworden sind; so treffen wir in Wien z.B. Freund, Grillparzer oder Dollfuß, aber auch die Kindesmörderin Josephine Luner.

Magris fordert - wie gesagt - die Leser heraus: er skizziert zahlreiche Episoden aus der europäischen Geschichte, gibt immer wieder Stichpunkte, Reizworte, nennt Namen, verleitet dazu, sich über das Gelesene hinaus auch mit sich selbst und der eigenen Einbindung in die mitteleuropäische Kultur zu beschäftigen. Und Magris, Professor für Germanistik an der Universität Triest, kennt sich wie kaum ein anderer in der Kulturgeschichte des mitteleuropäischen Raumes und besonders der Habsburgermonarchie aus. Bereits durch seine 1963 in Turin erschienene Dissertation Il mito absburgico nella letteratura austriaca moderna ${ }^{13}$ hat er in diesem Zusammenhang auf sich aufmerksam gemacht und damit in den deutschsprachigen Ländern heftigen Widerspruch hervorgerufen. Auch so manche Aussage in Donau

12 "Weltgeist" bei Hegel der "absolute Geist, insofern er sich in der Weltgeschichte als objektiver Geist verwirklicht". Bei Herder und Goethe eher "Inbegriff der alles belebenden Weltseele, deren göttliche Allgegenwart auch in den geschichtlich bedeutsamen Kulturleistungen der Menschheit zum Ausdruck gelangt. “ (Brockhaus Enzyklopädie, Bd. 20, 171974, s. 184).

13 Deutsch: Der habsburgische Mythos in der österreichischen Literatur, Salzburg 1966, vgl. Johannes Hösles Rezension: “Italiens zorniger Germanist", in: Neues Forum. Zeitschrifı für den Dialog 14, $1967,643-644$. 
wird nicht unwidersprochen bleiben, zeigt er doch an vielen Stellen recht deutlich den deutschen oder österreichischen "Volkscharakter" auf:

La storia tedesca, che ogni tanto si protende verso imperi universali e millenari, nasce spesso da una cornice provinciale, da un orizzonte municipale. (70)

Die deutsche Geschichte. die von Zeit zu Zeit zu universalen und tausendjährigen Reichen tendiert, nimmt häufig von einem provinziellen Rahmen, von einem kleinstädtischen Gesichtspunkt ihren Ausgang. (78)

La Mitteleuropa è terragnola, alpenstock e abiti di pesante panno verde, meticoloso ordine di erari e cancellerie: civiltà di chi ha perduto la familiarità con l'elemento liquido, con l'amnios materno e con le antiche acque originarie, e non si spoglia facilmente, perché senza giacca, confine, grado, distintivo e numero di registro si sente esposto e a disagio. (163)

Mitteleuropa bedeutet Landbewohner, Alpenstock und Anzüge aus schwerem grünen Tuch, die genaue Ordnung der staatlichen Kassen und Kanzleien. Es ist die Zivilisation dessen, dem die Vertrautheit mit dem flüssigen Element, mit dem Fruchtwasser und den uralten Gewässern des Ursprungs abhanden gekommen ist; man zieht sich nicht so leicht aus, denn ohne Anzug, Grenze, Rang, Distinktion und Registrationsnummer fühlt man sich exponiert und unbehaglich. (180)

... certo Vienna, come ma forse più di altre città, è anche il luogo di questi pettegolezzi e di queste astiose invadenze e indiscrezioni, perché è una grande città di provincia. (186)

...sicherlich ist Wien - vielleicht mehr als andere Städte - auch der Ort für solchen Klatsch und derartige mißgünstige und zudringliche Indiskretionen, da es eben eine große Provinzstadt ist. (205)

In diverse città della Germania e di altri paesi, le classi scolastiche si spopolano di bambini tedeschi e si riempiono di bambini turchi; l'occidente, che affida alla denatalità il proprio declino, reagisce con ansiosa superbia ai risultati del meccanismo sociale che esso stesso ha messo in moto. E' possibile che s'avvicini il momento in cui le diversità storiche, sociali e culturali, mostrino violentemente le difficoltà della convivenza; il nostro futuro dipenderà anche dalla convivenza; il nostro futuro dipenderà anche dalla nostra capacità di impedire che si inneschi questa mina dell'odio e che nuove battaglie di Vienna [M. spielt auf die Belagerung Wiens durch die Türken 1683 an] trasformino gli uomini in stranieri e innemici. (189) 
In verschiedenen Städten Deutschlands und anderer Länder füllen sich die Schulklassen, in denen die Zahl der deutschen Kinder immer weiter abnimmt, dafür mit türkischen Schülern; das Abendland, das seinen Untergang an den Geburtenrückgang delegiert, reagiert mit ängstlichem Hochmut auf die Auswirkungen eines gesellschaftlichen Mechanismus, den es selbst in Gang gesetzt hat. Es ist durchaus möglich, daß der Augenblick bevorsteht, da die geschichtlichen, gesellschaftlichen und kulturellen Unterschiede die Schwierigkeiten des Zusammenlebens auf gewaltsame Weise hervorkehren; unsere Zukunft hängt auch davon $a b$, ob wir fähig sein werden, zu verhindern, daß sich jener unterschwellige Haß entlädt und neue Schlachten vor Wien die Menchen in Ausländer und Feinde verwandeln. (208-209)

Danubio ist, wie der Text auf dem Schutzumschlag verspricht, ein "riesiges Mosaik“, ein Puzzle, das man beim Lesen letztlich selbst zusammensetzen muß. Ist die Neugier einmal geweckt - und sie zu wecken, versteht Magris meisterhaft —, ruht man erst, wenn man das letzte Mosaiksteinchen, das letzte Teilchen des Puzzles, am rechten Ort eingesetzt zu haben glaubt.

Mag das enzyklopädische Wissen des Autors manchen Leser auch gelegentlich in den Zustand ehrfürchtiger Bewunderung versetzen, so erlöst Magris ihn doch bald wieder davon, indem er menschliche Züge zeigt oder auf seine eigene Unwissenheit in bestimmten Dingen hinweist; so z.B. wenn er über das "Moidle-Schiff" spricht, auf dem Karl Alexander von Württemberg im Jahre 1719150 Frauen ins Banat schickte, wo sie die Unteroffiziere heiraten sollten, die sich nach dem Frieden von Passarowitz dort als Siedler niedergelassen hatten, und wenn er, Magris, hinzufügt: "Quella barca ... sarebbe un battello ideale per compiere questo viaggio, ... senz'alcuna fretta e anzi desiderando di non arrivare mai“ $(66)^{14}$, oder wenn er zugibt, er habe keine Hoffnung "di afferrare le leggi dell'economia e della scienza monetaria“ $(73)^{15}$.

Literarische Übersetzung ist kein einfaches Handwerk; und die Übersetzung dieses magnum opus von Claudio Magris erweist sich wegen der Z.T. oben angesprochenen Charakteristika der verwendeten Sprache als besonders schwierig. Aber fast ebenso schwierig ist das Vorhaben des Übersetzungskritikers, soll er doch, wenn er seine Tätigkeit ernsthaft betreibt, einer schlechten und von ihm kritisierten Übersetzung eine bessere gegenüberstellen ${ }^{16}$.

14 “Jenes Boot ... wäre ein ideales Schiff, um diese Reise zurückzulegen, ... ohne jede Eile, vielmehr mit dem Wunsch, niemals anzukommen." (73)

15 "Ich habe nicht die geringste Hoffnung, die Gesetze der Volkswirtschaft oder des Finanzwesens zu begreifen ...“ (81)

16 Vgl. u.a. Koller 1979: 209. Ich denke in diesem Zusammenhang an die Diskussion, die ich mit der Lektorin des Hanserverlages über den Passus "una specie di gioco dell'oca dell'amore“ (32) geführt habe. Der Übersetzer hat für gioco dell'oca den im Sansoni aufgeführten Terminus "Mensch-ärgeredich-nicht" (der allerdings der Sache nicht entspricht) verwendet, die Lektorin hat ihn gestrichen und "Gänsespiel" eingesetzt, eine Bezeichnung für ein Würfelspiel, das auch in Deutschland existiert, das aber - wie mir scheint - großen Teilen der deutschsprachigen Bevölkerung unbekannt ist. In der 
Der Kritiker, der beide Texte vergleicht, legt sich eine Werteskala zurecht, die von korrekt oder gut über akzeptabel bis zu ungenau oder einfach falsch reicht. Trotz dieses approximativen Schemas fällt es ihm häufig schwer, eine Entscheidung zu treffen (wie dem Phonetiker, der beim Abhören eines Tonbandes schwankt, ob er einen gehörten Vokal als offen oder geschlossen transkribieren soll). Wenn Magris von der Isis im Museum in Costanța am Schwarzen Meer sagt, sie habe "una bocca tumida e sensuale“ (411), so besteht sicher kein Zweifel darüber, daß sensuale mit 'sinnlich' zu übersetzen ist, aber ist 'mit wulstigen Líppen' eine korrekte Übersetzung für das Adjektiv tumido? Zweifellos spielen hier individuelle überlegungen und in der jeweiligen Gesellschaft verbreitete Vorstellungen von Ästhetik eine Rolle. Im Deutschen ist 'wulstig' wohl eher negativ besetzt, wodurch es im Text in Opposition tritt zu 'sinnlich'; wäre es da nicht besser gewesen, 'mit schwellenden' oder 'mit vollen Lipppen' zu schreiben? Der dadurch entstehende Pleonasmus verstärkt die Wirkung von 'sinnlich'. Das herkömmliche Bild von Isis, der höchsten Göttin der Ägypter wird daduch pervertiert, was, wenn man den Kontext betrachtet, gar nicht abwegig ist, denn in der Parade der im betreffenden Museum aufgestellten Götter befindet sich auch ein Apollo mit s̀chönem Frauenkopf, der "viel weiblicher und verführerischer [ist] als der der unweit aufgestellten Aphrodite“ (456) ${ }^{17}$. Der spanische Übersetzer entscheidet sich übrigens für henchido 'geschwollen' (im Spanischen würde für 'wulstig' - bezogen auf Lippen - eher grueso Verwendung finden), und in der französischen Fassung finden wir charnu, was 'fleischig' bedeutet. (471)

\section{Ein zweites Beispiel:}

"L'austriacità è arte della fuga ..." schreibt Magris auf S. 216. Nun kann fuga im Deutschen sowohl 'Flucht' als auch 'Fuge' im musikalischen Sinne bedeuten. Hier hat der Autor m.E. bewußt die Doppeldeutigkeit in Kauf genommen, ja geradezu gesucht und auf ein (wenn auch inhaltsleeres) Wortspiel ${ }^{18}$ zurückgegriffen. Gemeint ist hier jedoch nicht die Bach'sche "Kunst der Fuge", sondern die österreichische "Kunst der Flucht". Der spanische Text kann das Wortspiel aufgrund der in diesem Fall auftretenden semantischen Ähnlichkeiten übernehmen: "La austricidad

deutschen Fassung heißt es also: “eine Art Gänsespiel der Liebe“ (36). Kann man von den Lesern erwarten, daß sie sich mittels eines Lexikons "sachkundig" machen, wie es so schön heißt, oder wäre es nicht besser gewesen, die weniger exakte, dafür aber verständlichere Bezeichnung "Würfelspiel“ zu wählen? In Spanien ist das juego de la oca und in Frankreich das jeu de l'oie allgemein bekannt, so $\mathrm{daß}$ die Verwendung des Ausdruck in der spanischen (30) und der französischen Fassung (41) nicht überrascht.

17 “Un Apollo del primo secolo avanti Cristo è una bella testa di donna, molto più femminile e seducente di quella, poco distante, di Afrodite“ (411).

18 Vgl. H. Lausberg, Elemente der literarischen Rhetorik, München ${ }^{9} 1987$. Das Wortspiel stellt einen Verstoß gegen die perspicuitas, die "intellektuelle Verständlichkeit der Rede" dar (Lausberg, S. 50 u. 56), "kann aber (wie alle Fehler) infolge einer Lizenz (...) als Kunstmittel Verwendung finden...." (S. 56-57), in unserem Fall würde es sich um eine "wortinhalt-bedingte Äquivozität (...) zwecks ästhetischer Verfremdung" (S. 57) handeln. "Der Hörer wird zur eigenen Gedankenarbeit provoziert: er soll die Brücke zwischen dem Paradox und der gemeinten Bedeutung schlagen. Leistet der Hörer diese Arbeit, so freut er sich über seine eigene Intelligenz und ist so ein 'Gedanken-Komplize' des Autors." (S. 61). - Ein von mir um einen Übersetzungsvorschlag gebetener Österreicher plädierte übrigens für "Kunst des Sich-davon-schlängelns"! 
es el arte de la fuga" (188); und das in der französischen Fassung verwendete Wort fugue (250) läßt - zumindest umgangssprachlich - auch die Interpretation 'Seitensprung, Ausreißen' zu. Auch hier wird die Entscheidung sicherlich vom Bild, das der deutsche Übersetzer von der Sache - hier von Österreich - hat (oder nicht hat), beeinflußt. Es bleibt ihm an dieser Stelle nur die Möglichkeit, sich für eine Übersetzung zu entscheiden (eine Paraphrase, die das Wortspiel im Deutschen angemessen wiedergibt, ist nicht möglich). Er entscheidet sich - entgegen der Erwartung desjenigen, der Österreich und seine Geschichte kennt — für "Fuge": "Austriazität, das ist die Kunst der Fuge, das Vagabundentum, die Liebe zur Rast, während man auf eine Heimat wartet, die - wie Schuberts Wanderer sagt - immer gesucht, geahnt und nie gekannt werden wird" (239). François Bondy weist in seiner Rezension "Ein Dreitausend-Kilometer-Film" in der Süddeutschen Zeitung (1./2.10.88) auf diese Unstimmigkeit hin und erklärt, es sei 'mit 'fuga' wirklich Flucht gemeint, im Sinne von Hans Weigels Österreich-Buch 'Flucht vor der Größe'“19. Wenn ich mich diesem Urteil Weigels auch anschließen kann, so bin ich bezüglich seiner Aussage, dies sei das einzige Mißverständnis auf fast fünfhundert Seiten, doch anderer Meinung.

Seien wir uns darüber im klaren, daß man sich über einzelne Formulierungen, also über die "Mikrostruktur" der Übersetzung, in vielen Fällen streiten kann. Ausschlaggebend für die abschließende Bewertung einer literarischen Übersetzung bleibt letztlich die "ästhetische 'Gesamtökonomie'“, wie Katharina Reiß es nennt ${ }^{20}$. Zutreffend ist in diesem Zusammenhang die Äußerung Süskinds: "Der Originallautor hat aus dem vollen Besitz seiner Muttersprache geschrieben, also hat er Anspruch darauf, daß er auch in den vollen Genuß jener Ausdrucksmöglichkeiten gelangt, die unserer Sprache, und nur der unseren, eigentümlich sind." ${ }^{\text {21 Um einen }}$ äquivalenten Gesamteindruck zu erreichen, scheint es also z.B. auch gerechtfertigt, Normabweichungen des Autors in der Übersetzung an anderer Stelle nachzuvollziehen (sog. versetztes Aquivalent). Wenn Magris stellenweise wenig gebräuchliche Wörter verwendet, dann ist es durchaus legitim - mag es dem Kritiker gefallen oder nicht - wenn der Übersetzer an anderer Stelle Wörter, die im Italienischen geläufig sind, durch veraltete deutsche Wörter übersetzt. Gleiches träfe für den oben behandelten Fall des im deutschen nicht nachvollziehbaren italienischen Wortspiels zu.

Wenn man sich bei der Beurteilung der deutschen Fassung das Prinzip der ästhetischen Gesamtökonomie zu eigen macht, kann man durchaus zu dem von Bondy geäußerten Schluß kommen, daß die Übersetzung "diesem Buch im höchsten Maß gerecht wird". Ein genauer Vergleich von ausgangssprachlicher und zielsprachlicher Fassung läßt jedoch den Verdacht aufkommen, daß Bondy sich vornehmlich mit der deutschen Übersetzung beschäftigt hat, sonst hätte er eine Reihe von Unstimmigkei-

Styria Graz, ${ }^{3} 1978$.

Möglichkeiten und Grenzen der Übersetzungskritik, München 1971, 43.

21 W.E. Süskind, von der Vring, Georg: "Die Kunst des Übersetzens", in: Bayerische Akademie der Schönen Künste (Hrsg.): Die Kunst der Übersetzung, 8. Folge des Jahrbuchs Gestalt und Gedanke, München 1963, 9-39, Zitat S. 14. 
ten und sinnverändernden Abweichungen feststellen können. Sie aufzuzeigen ist nicht zuletzt Sinn und Zweck einer praktischen Übersetzungskritik.

Unter sinnverändernden Abweichungen ${ }^{22}$ verstehe ich:

1. Verstöße gegen die Logik; sie können vom Leser als solche identifiziert werden; es handelt sich also um Fehler, deren Vermeidung schon mittels einer genauen Einsicht in den beschriebenen Sachverhalt möglich gewesen wäre;

2. Übertragungen, die den vom Autor beschriebenen Sachverhalt zwar verfälscht wiedergeben, die jedoch beim Lesen nicht erkannt werden können, da sie weder gegen außersprachliche Determinanten noch gegen die - gegebenenfalls vom Kontext mitbestimmte - Logik verstoßen.

In allen Fällen setzen diese sinnverändernden Abweichungen eine mangelnde Kenntnis der Ausgangssprache und/oder falsche Interpretation der zu übersetzenden Strukturen voraus, von "Flüchtigkeitsfehlern" einmal abgesehen.

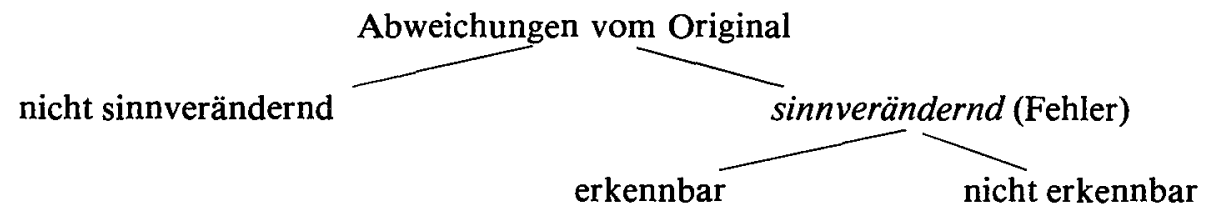

ad 1. (Verstoß gegen die Logik)

La casa è antica, la cucina risale al 1715 ; una vecchia ... invita bruscamente ... ad ascoltare ... un nastro che descrive il bruno focolare, gli utensili settecenteschi ... (24)

Das Haus ist alt, die Küche geht auf das 1715 zurück; eine alte Frau ... fordert brüsk dazu auf, ... einer Kassette zuzuhören, welche die dunkle Feuerstelle, die Küchenutensilien aus dem 17. Jahrhundert ... beschreibt. (27)

Spanisch: "los utensilios setecentistas ..." (24)

Französisch: "les ustensils $d u X V I I I^{e}$ siècle ..." (32)

22 Bei K. Reiß, Möglichkeiten und Grenzen der Übersetzungskritik, S. 22 "Unstimmigkeit der Aussage"; Herwig Kellner, "Übersetzen und Sinnklärung. Ein Beitrag zur Übersetzungskritik“, in: Lebende Sprachen 1964, 87-90, spricht von "Sinnwidrigkeiten": "Sinnwidrigkeiten fallen auch ohne Vergleich der Texte auf, von dem die meisten Kritiker aus Zeitmangel absehen“ (87). 
Korrekt muß es 'aus dem 18. Jahrhundert' heißen. Hier handelt es sich um einen fast durchgängigen Fehler des Übersetzers, der in den meisten Fällen von der Lektorin korrigiert, aber einige Male übersehen wurde.

[Der Autor befindet sich in Immendingen:]

Ma a Ulm, pochi chilometri più oltre... (32)

In Ulm dagegen, wenige Kilometer flußaufwärts... (36)

Natürlich muß es korrekt flußabwärts heißen. Der spanische Übersetzer entgeht dem Problem durch eine wörtliche Übersetzung: "Pero en Ulm, pocos kilómetros más allá..." (30); franz.: "Mais à Ulm, à quelques kilomètres en aval..." (41)

... la differenza ... fra la proprietà visibile di quel blu — che giunge alla corteccia cerebrale attraverso le onde elettromagnetiche e gli impulsi del nervo ottico - e ... $(19$

... die Differenz zwischen der sichtbaren Eigenschaft jenes Blaus - das durch elektromagnetische Wellen und mittels der Impulse des Sehnervs auf die Netzhaut gelangt - und... (22)

Schon sachlich kann vom Autor nur die 'Hirnrinde' (corteccia cerebrale), nicht die 'Netzhaut' gemeint sein; der Sehnerv verbindet das Auge mit dem Gehirn. Der spanische und die französischen Übersetzer haben aufgrund der lexikalischen Nähe von italienischer einerseits und spanischer und französischer Sprache andererseits keine Probleme mit dieser Passage: “... la diferencia entre ... la propiedad visible del azul - que llega a la corteza cerebral a través de las ondas electromagnéticas y los impulsos del nervio óptico - y...“ (20). “... la différence entre ... le caractère visible de ce bleu - qui parvient au cortex cérébral par des ondes électromagnétiques et l'influx du nerf optique - et..." (26)

ad 2. (sinnverändernde, in der Übersetzung nicht erkennbare Fehler)

... da adulto egli passa le ore serali a ricapitolare la sua infanzia e soprattutto quei momenti nei quali, da bambino chiudeva gli occhi beato mentre la madre preparava la cena. (90)

... seitdem er erwachsen ist, verbringt er die Abende damit, seine Kindheit zu rekapitulieren, und zwar insbesondere jene Momente, da er als kleines Kind glücklich die Augen schloß, während die Mutter das Mittagessen zubereitete. (99)

Span. und franz. korrekt cena, dîner. 


\section{Falsche Wortwahl}

... si guarda dal finestrino del treno che precipita nel paesaggio... (12)

... man schaut aus dem Fenster des Zuges, der die Landschaft beschleunigt...

... se mira por la ventanilla del tren que se precipita en el paisaje... (13)

... on regarde par la fenêtre du train qui fonce à travers le paysage (18) [foncer 'stürzen']

Übersetzungsvorschlag: ... der durch die Landschaft stürzt / hastet...

... il fiume scorre e scintilla nel sole come il fluire della vita... (15)

... der Fluß fließt und glitzert in der Sonne wie das verfließende Leben... (17)

... el río corre y brilla bajo el sol como el fluir de la vida... (16)

... le fleuve coule et scintille au soleil comme le cours de la vie... (22)

Übersetzungsvorschlag: ... wie der Lebensfluß / das Fließen des Lebens...

La paura, ha scritto Canetti, inventa dei nomi per distrarsi... (33)

Es ist die Angst, so schreibt Canetti, die in ihrer Zerstreuung Namen sucht und findet... (37)

Korrekt: 'zu ihrer Zerstreuung', spanisch: "El miedo, ha escrito Canetti, inventa nombres para distraerse..." (31), franz.: "La peur, a écrit Elias Canetti, invente des noms pour se distraire..." (43)

Céline ha visto il volto della Medusa, il vuoto che sta dietro il brulicare e il liquame della vita... (50)

Céline hat das Medusenhaupt gesehen, die Leere, die sich hinter dem Drägen und Fließen des Lebens verbirgt... (56)

Céline ha visto el rostro de la Medusa, el vacio que hay detrás de la confusión y de la podredumbre de las cosas... (45)

Céline a vu le visage de la Méduse, le vide qu'il y a derrière le fourmillement et le pus de la vie... (62) 
liquame ist 'Jauche', span. podredumbre, f., bedeutet 'Fäulnis, Verwesung'; dem spanischen Übersetzer ist an dieser Stelle offensichtlich nicht ganz wohl gewesen, denn er überträgt Magris Formulierung della vita in de las cosas, so daß es im Spanischen - etwas harmloser - 'Fäulnis der Dinge' heißt. Franz. pus 'Eiter'.

... il ministro Rudhard, un figlio di Passau, s'imbarcò nella sua città natale diretto ad Atene... (122-123)

... schiffte sich sein Minister Rudhard, ein Sohn Passaus, von seiner Heimatstadt aus direkt nach Athen ein; (135)

... el ministro Rudhard, hijo de Passau, se embarcó en sua ciudad natal hacia Atenas... (108)

... son ministre Rudhard, natif de Passau, embarqua aussitôt de sa ville d'origine pour Athènes... (144)

Im Spanischen wird diretto $a$ korrekt mit hacia, im Franz. mit pour wiedergegeben, im Deutschen müßte man wohl 'mit Ziel' oder 'nach' schreiben.

La grandezza del Lukács maturo consiste nella forza con la quale egli ha combattuto questo perdersi della vita nel nulla indistinto, strappandole con dura disciplina quei momenti significativi ... che altrimenti, affidati all'immediatezza spontanea, si dissolvono nell'assalto dispersivo delle cure. (293)

Die Größe des reifen Lukács besteht in der Kraft, mit der er diesen Verlust an Leben, dieses Vergehen in ein unbestimmtes Nichts bekämpft hat, indem er mit strenger Disziplin jene bedeutsamen Momente erfaßte..., die sonst, wenn sie einer spontanen Unmittelbarkeit überlassen bleiben, sich in einen Wirbel von Sorgen auflösen. (324)

La grandeza del Lukács maduro consiste en la fuerza con que combatió este perderse de la vida en la nada indiferenciada, arrancándole con dura disciplina los momentos significativos ... que, en caso contrario, confiados a la inmediatez espontánea, se disuelven en el asalto dispersivo de las preocupaciones. (253-254)

La grandeur de Lukács, dans sa maturité réside dans la force avec laquelle il a combattu cette déperdition de la vie dans un néant indistinct, en lui arrachant avec une discipline de fer ces moments significatifs ... lesquels, autrement, consacrés à l'immédiateté de ce qui se présente, se dissolvent sous les assauts tous azimuts des occupations quotidiennes... (339) 
Um den Sinn richtig wiederzugeben, $m u \beta$ man strappare hier mit 'entreißen' übersetzen, vgl. span. arrancar 'entreißen, entlocken', franz. arracher u.a. 'abtrotzen'.

Le snelle ed aguzze bandiere di ferro, sulle torri, si stagliano ferme e intrepide nel cielo e nel vento, cavalieri che attendono senza paura, nell'arena del torneo, un destino sconosciuto. (339)

Die schlanken spitzen Wetterfahnen auf den Türmen stehen fest und unerschrocken vor dem Himmel und gegen den Wind, Ritter, die auf dem Turnierplatz furchtlos einen unbekannten Gegner erwarten. (374)

Las esbeltas y agudas banderas de hiero, sobre las torres, se recortan firmes y intrépidas en el cielo y en el viento, caballeros que esperan sin miedo, en la arena del torneo, un destino desconocido. (292)

Les drapeaux de fer élancés et pointus, au sommet des tours, se détachent, immobiles et intrépides, dans le ciel, dans le vent - chevaliers qui attendent sans peur, dans la lice, un destin inconnu. (389)

In diesem Fall bleibt die Aussage des Satzes zwar im wesentlichen dieselbe wir können wohl davon ausgehen, daß sich der Übersetzer etwas dabei gedacht hat, als er destino statt mit 'Schicksal' mit 'Gegner' übersetzt hat - dennoch glaube ich, daß der Leser ein Recht darauf hat - wenn es sprachlich und stilistisch möglich ist -, die vom Autor intendierte Aussage auch in der Übersetzung wiederfinden zu können; und 'Schicksal' ist nun einmal nicht dasselbe wie 'Gegner'.

La lirica di Celan ${ }^{23}$ è un'estrema poesia orfica, un canto che scende nella notte e nel regno dei morti... (342)

Die Lyrik Celans ist bis zum Äußersten orphische Dichtung, ein Gesang, der in der Nacht, im Reich der Toten aufsteigt... (377)

La lírica de Celan es una extrema poesía órfica, un canto que desciende a la noche y al reino de los muertos... (294)

La poésie de Celan se situe aux limites extrêmes de l'orphisme, c'est un chant qui descend dans la nuit et dans le royaume des morts... (392)

korrekt: ... der in die Nacht und ins Reich der Toten hinabsteigt...

23 Paul Celan, "eigentlich Paul Antschel, Lyriker, "Czernowitz (Bukowina) 23.11.1920“, lebte in Paris, wo er 1970 Selbstmord beging. Seine “melodische Lyrik ist Geflecht ('Sprachgitter') von Chiffren, die über jeden Sinn hinaus ins logisch nicht Erfaßbare weisen. Anrufe in assoziativer Reihung sind in Sprache übersetzte Fugen. Manche seiner Gedichte enden im Verstummen vor dem Unsagbaren. Bes. bekannt ist sein Gedicht 'Todesfuge', die Elegie von der Ermordung der Juden im Konzentrationslager.“ (Brockhaus Enzyklopädie, Bd. 3. ${ }^{17} 1967$, S. 656). 
Diese Passage könnte bei entsprechender Kenntnis der griechischen Mythologie vom Leser auch ohne Rekurs auf das Original erkannt werden.

Graffiti a Ivanovo (380)

Sgraffiti in Iwanowo (421)

Graffiti en Ivanovo (327)

Les graffitis d'Ivanovo (435)

Sgraffiti kann hier wohl nicht gemeint sein, dabei handelt es sich nach einer Definition des Dudens nämlich um "Fassadenmalerei, bei der die Zeichnung in die noch feuchte helle Putzschicht bis auf die darunter liegende dunkle Grundierung eingeritzt wird", vielmehr geht es, was aus dem Kontext deutlich wird, um "Schmierereien", also "Graffiti".

Worter und Sachen (außersprachliche Determinanten, Sachbezug ${ }^{24}$, Ortsbezug)

Per i contadini slovacchi c'erano le drevenice, le capanne o piccole case di assi di legno cementate da paglia e letame secco. (236)

Für die slowakischen Bauern gab es die drevenice, Hütten oder kleine Häuser aus Brettern, die mit Stroh und getrocknetem Mist verputzt wurden. (260)

A los campesinos eslovacos les estaban destinadas las drevenice, las cabañas o casitas con vigas de madera cimentadas mediante paja y estiércol seco. (205)

Pour les paysans slovaques il y avait les drevenitsas, ces cabanes ou maisonnettes en planches et en torchis de paille et de fumier séché. (272)

Einige Zeilen weiter heißt es: "per secoli, gli slovacchi sono stati un popolo ignorato, l'oscuro sostrato e tessuto del loro paese, simile a quella paglia e a quel letame secco che tiene insieme le drevenice (236) - "über Jahrhunderte sind die Slowaken ein unbeachtetes Volk gewesen, ein dunkler Nährboden ihres Landes, ein Stoff ähnlich jenem aus Stroh und getrocknetem Mist, der die drevenice zusammenhält", es kann sich also wohl nicht nur um den Putz handeln, sondern vielmehr um das verwendete Bindemittel. Der franz. Text verwendet einen Fachterminus: torchis 'Strohlehm' ("Terre grasse argileuse, malaxée avec de la paille hachée ou du foin (utilisée pour lier pierres d'un mur, pour former le hourdis d'une construction en colombage)“, Le Petit Robert), der den vom Autor gemeinten Sachverhalt ziemlich genau wiedergibt.

${ }_{24}$ Vgl. K. Reiß. Möglichkeiten und Grenzen der Übersetzungskritik, Kap. 4, 69-88. 
Falscher Bezug durch fehlerhafte Interpretation des Relativpronomens (Verwechslung der grammatischen Person)

... il Mulato cui una Spagnola regala un azzimato Morisco... (32)

... der Mulatte, den eine Spanierin einem aufgeputzten Morisco schenkt... (36)

cui entspricht al quale, also: der Mulatte, dem eine Spanierin einen aufgeputzten Morisco schenkt...

Im Spanischen und Französischen ist die Konstruktion richtig erkannt und übersetzt worden:

... el Mulato al que una Española regala un adornado Morisco. (30)

... le Mulâtre à qui une Espagnole donne un adorable petit Maure. (41)

\section{Falscher Bezug durch fehlerhafte Interpretation der Verbform}

... come diceva Curtius, "la storia non conosce le origini di nessun popolo“, perché essa non esiste ma è la storiografia che la crea e la produce, ponendo e investigando la sua questione. (391)

... "die Geschichte kennt keines Volkes Anfänge", schreibt Curtius, weil sie nämlich gar nicht existieren; es ist vielmehr die Historiographie, die sie schafft und hervorbringt, indem sie dieses Problem stellt und untersucht. (434)

... como decía Curtius, "la historia no conoce los orígenes de ningún pueblo“, porque no existe y es la historiografía quien la crea y la produce, planteando y investigando su cuestión. (336)

... comme disait Curtius, "l'Histoire ne connaît les origines d'aucun peuple“, parce que cela n'existe pas; c'est une création et une production de l'historiographie, qui pose la question puis se livre à des recherches pour y répondre. (448)

Korrekt: ... weil sie [nämlich die Geschichte] nicht existiert. Im Franz. ist der Bezug nicht eindeutig, da die Pronomina essa. und la durch das unpersönliche cela bzw. $c(e)$ übersetzt werden, die sich sowohl auf histoire als auch auf peuple beziehen können.

\section{Unkorrekte Wiedergabe des Genus verbi}

L'epicità agraria imprime uno stile sovrapersonale, che esclude ogni vanitosa soggettività. (323) 
Das ländliche Epos ist von einem überpersönlichen Stil geprägt. (356)

La epicidad agraria imprime un estilo sobrepersonal, que excluye cualquier vanidosa subjetividad. (279)

L'épique agraire imprime un style qui dépasse l'individu, qui exclut toute subjectivité vaniteuse. (371)

Magris verwendet im Italienischen das Aktiv, während in der deutschen Übersetzung das Passiv steht; dadurch erfolgt eine Vertauschung von Agens und Patiens.

Korrekt: Das ländliche Epos [besser: bie ländliche Epizität] prägt einen überpersönlichen Stil.

Trotz der relativ zahlreichen sinnverändernden Fehler, die allerdings größtenteils nicht augenfällig sind, wird die Atmosphäre, die Magris im Original erzeugt, auch in der deutschen Fassung im großen und ganzen vermittelt. Dennoch merkt man an verschiedenen Stellen, daß es sich um eine Übersetzung handelt, z.B. wenn der Übersetzer zu sehr bemüht ist, im Deutschen die italienische Syntax beizubehalten, oder wenn sich in die Übersetzung unbeabsichtigt syntaktische Interferenzen einschleichen, was dann linkisch oder archaisch klingt:

Nei suoi romanzi e soprattutto nei suoi racconti, molti dei quali scritti in quelle stanze, Stifter indaga... (135)

In seinen Romanen und insbesondere in seinen Erzählungen, deren viele er in ebendiesen Zimmern geschrieben hat, erforscht Stifter... (149)

Bei der Einsicht in die Rohübersetzung, die mir von der Lektorin des Hanserverlages ermöglicht wurde, ist mir eine Reihe von Übersetzerfehlern aufgefallen, die in die Kategorie "Stilblüte" eingeordnet werden könnten, die aber von der Lektorin korrigiert wurden. In der gedruckten deutschen Fassung tritt nur ein solcher Fall auf:

Anche le stanze dell'Osteria al Cimitero dei Senzanome fanno pensare a una piacevole sosta nel cammino, a camerette ospitali. (203)

Auch die Zimmer des Gastwirtes beim Friedhof der Namenlosen lassen einen angenehmen Aufenthalt und gastfreundliche Zimmermädchen erwarten. (224)

También las habitaciones de la Posada en el Cementerio de los Sinnombre hacen pensar en una agradable parada en el camino, en cuartitos acogedores. (177) 
Même les chambres de l'Hôtel du Cimetière des anonymes évoquent une halte agréable au voyageur, des chambrettes accueillantes. (234)

Korrekt müßte es im Deutschen Zimmerchen heißen, hier liegt eine Verwechslung mit cameriera vor. Im Span. und Franz. wird das ital. Wortbildungsmuster mittels Diminutivsuffix nachvollzogen: span. cuarto $\rightarrow$ cuartito, franz. chambre wird - vielleicht etwas ungewöhnlich, aber durchaus möglich - zu chambrette.

Der Vergleich des italienischen AS-Textes mit verschiedenen zielsprachlichen Fassungen untermauert einmal mehr die Feststellung, daß eine Übersetzung aus einer romanischen Sprache in eine andere auf wesentlich weniger Schwierigkeiten stößt als die Übersetzung ins Deutsche. Abgesehen davon, daß die verschiedenen romanischen Sprachen zum großen Teil über dasselbe Lexikon verfügen, kann meistens auch die Syntax der AS in der ZS beibehalten werden, während im Deutschen oftmals - sei es aus grammatikalischen Gründen, sei es aufgrund stilistischer Überlegungen - bestimmte Satzteile an ganz anderer Stelle im Satz stehen (oder stehen müssen) als im Italienischen (sowie im Spanischen und Französischen), wie im folgenden Beispiel das Prädikat des Objektsatzes, das durch das Objekt und eine Apposition vom Prädikat des Hauptsatzes getrennt ist.

Gli scrittori tedeschi cercavano di conciliare la fedeltà all'autonomia transilvana col germanesimo e con la corona absburgica, con la devozione a Francesco Giuseppe quale imperatore d'Austria e non quale re d'Ungheria. (336-337)

Die deutschen Schriftsteller waren darum bemüht, die Treue zur transsylvanischen Autonomie mit dem Deutschtum und der Habsburger Krone, mit der Verehrung Franz Josephs - nicht als König von Ungarn, sondern als Kaiser von Österreich $-z u$ versöhnen. (372)

Im Span. und Französischen kann die italienische Konstruktion übernommen werden:

Los escritores alemanes intentaban conciliar la fidelidad a la autonomía transilvana con el germanismo y con la corona habsbúrgica, con la devoción a Francisco José como emperador de Austria y no como rey de Hungría.

Les écrivains allemands cherchaient à concilier leur fidélité à l'autonomie de la Transylvanie avec le germanisme, la couronne des Habsbourg et la dévotion à François-Joseph en tant qu'empereur d'Autriche et non comme roi de Hongrie. (386-387)

Ebensowenig wie sich die Übersetzungskritik darauf beschränken darf, nur eine Zusammenstellung von Fehlern zu liefern, sondern vielmehr bessere Lösungen anbieten soll, ebensowenig dürfte sie sich damit zufrieden geben, nur zu kritisieren; zu einer "ehrlichen" (also möglichst objektiven) Übersetzungskritik gehört es auch, gu- 
te Lösungen für besonders problematische Texstellen hervorzuheben. In der Tat finden sich auch in der deutschen Fassung von Magris' Buch eine Reihe von solchen guten Lösungen:

\section{Fehlendes Lexem im Deutschen}

Per fortuna esiste la teleselezione. (202) (224)

Glücklicherweise kann man von den Zellen aus Ferngespräche führen.

Im Deutschen fehlt ein Lexem, das dem italienischen teleselezione entspricht. Die deutsche Lösung scheint mir besser gelungen zu sein als die spanische, die zwar die syntaktische Konstruktion beibehält, aber den Sachverhalt weniger genau wiedergibt als das Deutsche:

"Menos mal que existen las conferencias interurbanas" (177); durch die Ergänzung von posibilidad 'Möglichkeit' wäre der Übersetzer dem ital. teleselezione, nähergekommen. Die franz. Übersetzung trifft den Sachverhalt der italienischen Aussage ähnlich genau wie die deutsche:

"Heureusement qu'on a inventé le téléphone automatique" (234).

\section{Semantisches Problem}

Il movimento agrario bulgaro ha avuto un carattere aperto e progressivo, come rivela la politica del suo massimo leader, Stamboliski, e non conosce certo $\mathrm{i}$ toni regressivi e fascisti presenti in altri movimenti verdi, ad esempio negli "uomini verdi“" sognati da Codreanu... (373)

Die bulgarische Agrarbewegung war von offenem und fortschrittlichem Charakter, wie die Politik Stamboliskis, ihres bedeutendsten Führers, bezeugt, und kennt nicht jene regressiven und faschistoiden Züge, wie sie sich bei anderen bodenständigen Ideologien und Bewegungen finden, so zum Beispiel bei den von Codreanu, ... erträumten "grünen Menschen". (412-413)

El movimento agrario búlgaro ha tenido un carácter abierto y progresista, como revela la política de su máximo leader, Stamboliski, y está claro que desconoce los tonos regresivos y fascistoides presentes en otros movimientos verdes, por ejemplo en los "hombres verdes" soñados por Codreanu... (321)

Ce mouvement agraire bulgare a eu un caractère ouvert et progressiste, comme le révèle la politique de son principal leader, Stamboliski, et ignore totalement les nuances réactionnaires et fascistes présentes dans d'autres mouvements agrariens, comme par exemple celui des "hommes verts" rêvé par Codreanu... (427) 
movimenti verdi darf hier nicht mit 'grüne Bewegungen' übersetzt werden. So wie beim deutschen Leser falsche Assoziationen geweckt würden ${ }^{25}$, ist das spanische movimientos verdes nicht sehr glücklich, auch wenn es sich um eine wörtliche Übersetzung handelt, da der Ausdruck auch in Spanien mit der ökologischen Bewegung in Beziehung gebracht wird, selbst wenn dieser längst nicht die Bedeutung zukommt wie in der Bundesrepublik. Zweifellos ist sich auch Magris der Doppeldeutigkeit seiner Formulierung bewußt gewesen; allerdings handelt es sich hier nicht - wie oben im Falle von fuga - um ein einfaches Wortspiel; er muß sich bewußt sein, daß die Nennung von movimenti verdi im Zusammenhang mit toni regressivi e fascisti unter Umständen zu unrichtigen Schlußfolgerungen von Seiten der Leser führt.

Erleichtert wurde die Arbeit des Übersetzers - das soll auch nicht verschwiegen werden - durch die Kooperation des Autors selbst, der beispielsweise eine 21seitige Liste von nicht italienischen Termini bzw. Originalzitaten erstellt und an der bereits von der Lektorin überarbeiteten Rohübersetzung noch einige Änderungen vorgenommen hat. Dieses Zusammenspiel von Autor, Lektorat und Übersetzer war letztlich Gewähr dafür, daß die deutsche Fassung - insgesamt gesehen - ebenso mit Gewinn gelesen werden kann wie das Original.

Ich bin der Auffassung, daß der praktischen Übersetzungskritik innerhalb der Sprachwissenschaft zu wenig Aufmerksamkeit gewidmet wird, und daß eine engere Zusammenarbeit zwischen Linguisten und Verlagen für beide durchaus von Nutzen sein kann.

"L'esistenza dello scrittore“ sagt Magris S. 23, indem er Della Casa ${ }^{26}$ zitiert, "è uno stato di guerra". - "Die Existenz der Schriftstellers ... ist ein Kriegszustand" (27). Das gilt nicht weniger für den Übersetzer, und der Übersetzungskritiker beobachtet beide aus sichere Entfernung!

25 Ich erinnere an die vor wenigen Jahren in der Bundesrepublik geführte stark emotionalisierte Diskussion, nachdem den Grünen von CDU-Politikern faschistische Ansichten vorgeworfen worden waren.

26 Giovanni della Casa, ital. Humanist, 1503-1556. 


\section{Danubio CLAUDIA MAGRISA. KRITIČNI PRETRES PREVODOV}

Avtor analizira prevode sodobnega italijanskega literarnega dela Danubio Claudia Magrisâ, tržaškega pisatelja in profesorja nemške književnosti na Univerzi v Trstu, in primerja italijanski original $s$ prevodi v nemščino, španščino in francoščino. (Delo je bilo prevedeno tudi v slovenščino.) Največ odstopanj kaže prevod v nemščno: ugotovitev ni presenetljiva, saj je prevajanje v sorodne jezike v nekaterih pogledih manj zahtevno. Naveden je primer prevoda igrice il gioco dell'oca: ta igra s kockami je $v$ romanskih deželah dobro znana in potemtakem prevod ne predstavlja težav; $v$ nemških deželah pa očitno ne in tako je prevajalec, dokaj neustrezno, poimenoval igro s "Človek, ne jezi se!" Avtor predlaga za take primere bolj splošne izraze, npr. "igra s kockami".

Ko kritik tehta prevod, ga primerja z izvirnikom (te možnosti seveda bralec nima, razen pri redkih dvojezičnih izdajah) in si ustvari neko vrednostno lestvico: dobro/pravilno - sprejemljivo - neustrezno - napačno.

Prevajanje ima v sebi veliko pasti. Včasih je napaka očitna, tam npr. ko se v nemščino italijanski Settecento prevaja s "17. stoletje“ (prevajalec v romanske jezike take napake ne bi nikoli zagrešil). Nevarna past so semantični premiki. Ko govori Magris o zelenih gibanjih, misleč na fašistična gibanja v Romuniji, sta se tej nevarni čeri prevajalca $v$ nemščino in francoščno modro izognila (bodenständigen Ideologien und Bewegungen; mouvements agrariens): upravičeno, misli avtor, saj ima danes izraz gibanje zelenih, ipd., čisto drugačno konotacijo. 